\title{
Perceptions of Domestic Robots' Normative Behavior Across Cultures
}

\author{
Huao Li \\ hul52@pitt.edu \\ University of Pittsburgh \\ Pittsburgh, PA
}

\author{
Stephanie Milani ${ }^{*}$ \\ stemila1@umbc.edu \\ University of Maryland, Baltimore \\ County \\ Baltimore, MD
}

\author{
Vigneshram Krishnamoorthy \\ vigneshk@andrew.cmu.edu \\ Carnegie Mellon University \\ Pittsburgh, PA
}

\author{
Michael Lewis \\ ml@sis.pitt.edu \\ University of Pittsburgh \\ Pittsburgh, PA
}

\author{
Katia Sycara \\ katia@cs.cmu.edu \\ Carnegie Mellon University \\ Pittsburgh, PA
}

\begin{abstract}
As domestic service robots become more common and widespread, they must be programmed to efficiently accomplish tasks while aligning their actions with relevant norms. The first step to equip domestic robots with normative reasoning competence is understanding the norms that people apply to the behavior of robots in specific social contexts. To that end, we conducted an online survey of Chinese and United States participants in which we asked them to select the preferred normative action a domestic service robot should take in a number of scenarios. The paper makes multiple contributions. Our extensive survey is the first to: (a) collect data on attitudes of people on normative behavior of domestic robots, (b) across cultures and (c) study relative priorities among norms for this domain. We present our findings and discuss their implications for building computational models for robot normative reasoning.
\end{abstract}

\section{CCS CONCEPTS}

- Computing methodologies $\rightarrow$ Artificial intelligence; $•$ Computer systems organization $\rightarrow$ Robotics; $\bullet$ Human-centered computing $\rightarrow$ Human computer interaction (HCI).

\section{KEYWORDS}

human-robot interaction; service robots; cross-culture study; moral decision making; machine ethics

\section{ACM Reference Format:}

Huao Li, Stephanie Milani, Vigneshram Krishnamoorthy, Michael Lewis, and Katia Sycara. 2019. Perceptions of Domestic Robots' Normative Behavior Across Cultures. In AAAI/ACM Conference on AI, Ethics, and Society (AIES '19), fanuary 27-28, 2019, Honolulu, HI, USA. ACM, New York, NY, USA, 7 pages. https://doi.org/10.1145/3306618.3314251

*Work done as an intern at Carnegie Mellon University.

Permission to make digital or hard copies of all or part of this work for personal or classroom use is granted without fee provided that copies are not made or distributed for profit or commercial advantage and that copies bear this notice and the full citation on the first page. Copyrights for components of this work owned by others than the author(s) must be honored. Abstracting with credit is permitted. To copy otherwise, or republish, to post on servers or to redistribute to lists, requires prior specific permission and/or a fee. Request permissions from permissions@acm.org.

AIES '19, January 27-28, 2019, Honolulu, HI, USA

(c) 2019 Copyright held by the owner/author(s). Publication rights licensed to ACM. ACM ISBN 978-1-4503-6324-2/19/01...\$15.00

https://doi.org/10.1145/3306618.3314251

\section{INTRODUCTION}

With the development of robotics technology, robots' set of responsibilities has expanded from manufacture, military and rescue to include diverse roles in our daily life, such as being a personal assistant, a caretaker for children, and a housekeeper. These tasks can be programmed to have explicit rewards purely based on the accomplishment of the assignment; however, when these robots must interact with humans, there is usually no explicit reward function or correctness measure for their behavior. Instead, the appropriateness of an action depends on external factors - such as the physical environment and the social context - and internal, inferred factors - such as the users' personality, prior experience, and cultural background.

One important factor that informs the appropriateness of actions is the set of norms within a particular culture. Norms, such as prohibitions, permissions, obligations, are the socially agreed upon guidelines of behavior which are acknowledged by most of the members of a community [3]. The social science literature (e.g. [17] have reported that norm activation is dependent on environmental and social context. Additionally, we claim that determination of priorities among different norms also depends on the culture of the society where the robot operates. Each culture has its unique set of norms due to historical and socio-political reasons. Some cultures have strict social norms and severe punishment for norm violations, while others have weaker constraints and higher tolerance to individual discretion [6]. These social norms not only regulate the behavior selection of everyone in the society, but also help predict other agents' actions to improve collaboration [17]. Thus, to understand and design for effective interactions of robots with humans, it is critical for the robots to incorporate socio-cultural norms in their decision-making process in performing their various tasks.

The normative reasoning of intelligent agents, also called machine ethics [20], has attracted increasing attention in the community in recent years. Generally, representative works mainly concentrate on two types of problems. First is considering thought experiments such as the trolley problem to examine the appropriate moral decision an autonomous agent should make [2,18]. Second is focusing on task-specific environments - like judging performance in a game [9] or navigating indoors [23] - to evaluate the normative role of robots in social interactions. Recently, researchers are calling 
for more realistic scenario settings, as well as a general normative framework $[1,4,12]$. The first step towards these goals is to discover people's opinions about what norms robots should adhere to in specific situations and contexts, and how robots should prioritize their norms. However, to the best of our knowledge, currently there are no surveys that ask participants for their views on these issues. The work presented in this paper fills this gap. Additionally, our work studies these issues across two cultures to determine whether cultural difference exist. The domain of application of our survey is domestic service robots.

\section{RELATED WORKS}

Previous research in human-robot interaction in a domestic setting mainly focuses on the general acceptance and representation people have towards future domestic robots. Relevant works examine the correlation between age and the degree of autonomy granted to the domestic robot [25], the nature of expected tasks of domestic robots at home [26], and the influence of utilitarian attitudes and social norms on the acceptance of domestic robots [5]. However, the aforementioned works ask general questions. As has been found in the literature (e.g. [17]) norm activation is context-specific and therefore surveys would need to ground their questions to specific, detailed, realistic scenarios.

Beyond the micro context where the interactions occur, the macro socio-culture environment needs to be considered when modeling the norm framework. Existing work found that individuals from different cultures differed in their perceptions of social robots along the axes of trustworthiness [16], appearance [14], and politeness [24]. Literature has adopted culture dimensions such as Hofstede's [8] to examine the cultural factors in people's attitude and acceptance towards robots [7, 16, 21]. Although Hofstede's dimensions provide a general picture of how people behave across cultures, it ignores the impact of social contexts when determining an individual's behaviors in specific situations. As proposed by recent research $[10,15]$, it is necessary for cross-cultural studies to consider cultural syndromes that incorporate the interaction of cultures, individuals, and contexts. We incorporate cultural syndromes [15] as well as consideration of tight vs loose cultures [6] since they make particular predictions about norm compliance across cultures.

\begin{tabular}{|c|c|}
\hline Name & Description \\
\hline \hline Safety & Protect human from danger \\
Consideration & Consider human's feelings \\
Privacy & Protect human's privacy \\
Security & Secure sensitive information \\
Efficiency & Finish the given task efficiently \\
Compliance & Obey social rules \\
Command & Follow owner's command \\
Accommodation & Accommodate human's behavior \\
Honesty & Tell the truth \\
Loyalty & Maximize owner's interest \\
\hline
\end{tabular}

Table 1: Norm List

\section{METHOD}

\subsection{Methodological Design}

Previous literature on the general attitudes of people towards robots $[11,22,25,26,29]$, have determined various issues, such as the capability of the robot to finish a given task, invasion of privacy that may arise due to the robot's presence, and potential danger to safety.

Inspired by this literature and literature on ethics, we considered a (not necessarily exhaustive) list of general norms (Table 1) that arise in domestic robot interactions with humans. This list provided a guideline for studying people's attitudes and preferences on the acceptable normative behaviors of domestic robots.

We distinguish between the following terms when discussing the survey:

- A scenario, or situation refers to the description of the setting where the robot must choose how to respond.

- An option refers to a possible action that the robot could take in a specific scenario, which indicates the priority of a certain norm.

- Context refers to the physical and social environmental features which are manipulated within a scenario.

- Norms are the principal expectations, prohibitions and permissions on the behaviors of domestic robots.

To illustrate the notions of scenario, context, and potential normative actions of the robots, we provide below sample scenario, referenced throughout as the warning of allergy scenario:

Imagine that you are hosting a dinner at your home. You/your robot finish cooking and are about to serve a dish to your friends/family members. Your robot detects that one of the guests may be slightly/severely allergic to an ingredient in the dish through the conversation between them. He/she didn't provide allergy information in advance. You and the guests are midconversation. Please rate your opinion toward the following statements about robot's behavior.

- The robot should interrupt the ongoing conversation and tell everyone about the allergy situation.

- The robot should discreetly inform you (the robot owner), but not anyone else.

- The robot should do nothing.

To determine people's priority between norms, we designed scenarios, referred to as dilemmas, where several norms (some of them conflicting) could be simultaneously activated so the robot would need to choose the most appropriate action. For example, in the warning of allergy scenario, the conflicting norms are ensure safety, consider people's feelings, and obey social rules. For the robot, obeying the "ensure safety" norm could be achieved by interrupting the conversation immediately, informing everyone about the allergy; however, as a consequence, it may embarrass the hosts/owners and guests. An alternative action of the robot could be to discreetly inform the owner of the situation, but not anyone else. Doing so considers people's feelings, but sacrifices the opportunity to immediately warn the guest. The third option, "do nothing due to table manners", emphasizes compliance to social rules like do not 


\begin{tabular}{|c|c|c|c|c|}
\hline Scenario & Location & $\begin{array}{c}\text { Context } \\
\text { Characteristic }\end{array}$ & Consequence & Norms \\
\hline Warning of Allergy & Dining Room & Family/Friends & $\begin{array}{l}\text { Severe/Mild allergy, } \\
\text { Responsibility }\end{array}$ & $\begin{array}{l}\text { Safety, Consideration, } \\
\text { Compliance }\end{array}$ \\
\hline Entering Room & Bathroom/Bedroom & Fire/Burst Pipe & Drenched/Burned & $\begin{array}{l}\text { Safety, Privacy, } \\
\text { Compliance }\end{array}$ \\
\hline Ordering Groceries & Owner's office & $\begin{array}{l}\text { Payment Information/ } \\
\text { Shopping History }\end{array}$ & Unwanted Disclosure & Security, Efficiency \\
\hline Encountering Human & Hallway & $\begin{array}{l}\text { Elderly/Disabled, } \\
\text { Number of people }\end{array}$ & Injury & $\begin{array}{l}\text { Compliance, Safety, } \\
\text { Accommodation }\end{array}$ \\
\hline Judging Outfit & Party & Friends/Strangers & Embarrassment & Honesty, Consideration \\
\hline Reporting Evidence & Sidewalk & $\begin{array}{l}\text { Elderly/Strangers, } \\
\text { Responsibility }\end{array}$ & Liability & $\begin{array}{l}\text { Honesty, Loyalty, } \\
\text { Command }\end{array}$ \\
\hline
\end{tabular}

Table 2: Scenario List, possible values of a contextual variable are divided by slashes, different variables or norms are divided by commas. Norms in each scenario correspond to optional actions in order.

interrupt ongoing conversations. Surveying people about their preferences regarding those options enables us to determine both the norm priorities and the severity of the conflict between norms in the given scenarios.

For each scenario, participants were required to independently rate their opinion towards each presented option on a scale of 1 (strongly disagree) to 7 (strongly agree). The participants' answers within a particular scenario might be mutually exclusive (agree on one option and disagree on the other options), or not (agree or disagree on both options to different degrees). For example, a participant may rate 7 on the "interrupt option" and 1 on the "discreetly inform" option, indicating a strong participant preference of ensuring the guest's safety over embarrassing the hosts. Another participant may rate 6 on the "discreetly inform" option and 5 on the "interrupt option", which shows that they value those two options and the corresponding norms to similar degrees.

To measure the influence of context on norm activation, we manipulated key words in the scenario descriptions to present the scenario with different context. In general, context varies in three different aspects: 1) location of the interaction, 2) characteristics of those in the scenario, including age, number of people, and their relationship to the domestic service robot owner, and 3) the consequences of robot's behavior, which depends on the severity of consequences. A list of scenarios, corresponding contexts, and norms discussed in this paper is shown in Table 2. In the allergy case, we changed three contextual variables: 1 ) the owner or robot is serving the dish (responsibility), 2) the guests are friends or family (relationship), and 3) the allergy is slight or severe (consequences). Each participant read a randomly chosen version of this scenario. For example, one participant may read the version where the consequence of allergy is mild, but the other may read the one where it is severe. By comparing the answers between participants who read different versions of the same scenario, we determined the influence of contexts on the priority of norms.

\subsection{Questionnaire Design}

We designed 15 scenarios capturing distinct norm conflicts. To help participants better understand the scenario, we provided a detailed description and picture for each scenario. Each participant read all of the 15 scenarios; however, the context of each scenario randomly varied between subjects. For example, the allergy case had three contextual variables, each with two different levels that were changed: responsibility, relationship, and the severity of consequence. This gives rise to $8\left(2^{*} 2^{*} 2\right)$ different versions of this specific scenario that were evenly distributed to participants. The number of versions for each scenario ranged from 1 to 8 depending on the number and levels of contextual variables, and they were evenly distributed among participants. For each scenario, participants were asked to rate their opinion towards each of the (2 or 3 ) given options on a 7-point Likert scale (1- strongly disagree and 7- strongly agree). Before the main section, we included a section to collect demographic information - including gender, age, employment status, educational background, familiarity with robots, and primary source of information for knowledge of robots. After the context section, participants were asked two questions about their general acceptance and purchase tendencies about the robot described in the survey. The survey was originally designed in English, and was then translated to Chinese for running in mainland China. The Chinese version of the survey was then back-translated to English and examined by researchers to ensure equivalence.

\subsection{Data Collection}

To combat the limitations of traditional survey methods, such as less representative sampling [19], we conducted both versions of the survey using online platforms. The English version was built on Qualtrics.com, and published on Amazon Mechanical Turk for workers to access. The Chinese version ran on a similar platform, Wjx.cn, which provides both online questionnaires and sampling services in China. Each participant was paid \$1 to finish the 15minute survey.

\subsection{Participants}

To ensure that the participants from online platforms were from two target countries, we filtered the submissions by their IP address. After filtering, we were left with 481 samples from the MTurk 
American participants and 648 samples from the Wjx Chinese participants. After removing data of participants that did not finish the survey, or those who blindly clicked and had an extremely short finishing time, and those who answered the attention check question incorrectly, we were left with 301 (American) and 435 (Chinese) valid submissions. In the sampling population of the American survey, the average age was $40.6(\mathrm{SD}=11.6)$ and the gender ratio was about balanced ( $49.28 \%$ male, $50.71 \%$ female). In the Chinese version of the survey, the average age and gender ratio of the sampling population was $30.9(\mathrm{SD}=7.9)$ and $36.47 \%: 63.53 \%$ (male:female).

\section{RESULTS}

The survey results consisted of ratings of 39 options from 15 scenarios, and was influenced by both the individual differences of participants (e.g. age and cultural background) and the contextual variables of the scenarios (e.g. locations, characteristics, and consequences). Because participants gave independent ratings to each option based on their preferences, the given responses to the scenarios may or may not be mutually exclusive. Thus, when analyzing the data, the first-fold comparison is between options which explicitly reveal participants' preferences with respect to the conflicting norms. The second-fold comparison is a betweensubject comparison of factors, including cultures, contexts, and their interaction effects. Repeated measures ANOVAs were used to statistically evaluate the two-fold comparisons mentioned above, since norm ratings are measured within-subject while culture and context are between subject variables. When it comes to the pairwise comparison on a certain level of variable, one-way ANOVAs were employed. For variables with more than two levels, post-hoc tests using LSD method were reported. Due to the page limitation, the following results section only provides a preliminary analysis on the most informative findings for 6 out of the 15 scenarios. Descriptions of scenarios and options have been condensed.

\subsection{Encountering Human in Hallway}

Imagine your domestic robot is taught the concept of moving on the right side of the hallway. What should the robot do when it is moving down the hallway on the right side, while an (elderly/disabled) person is walking towards it on the same side?

- Stay to the right-hand side of the hallway to obey social rules.

- Move out of the way to the left-hand side of the hallway to maintain the safety of the human.

- Move out of the way to the left-hand side of the hallway to accommodate the human's behavior.

Generally, participants from both countries, as shown in Fig. 1, preferred the robot to give way to the person who is breaking the rule of walking to his/her right side of the hallway $(F(2,1468)=$ $487.94, p<.001, \eta_{p}^{2}=40.0 \%$, post-hoc test $\left.p s<.001\right)$. However, we found a significant cultural difference in this scenario: $\mathrm{CN}$ participants agreed more $(3.34 \pm 1.62)$ on the "stay on right-hand side" option compared with US samples $(2.65 \pm 1.65, F(1,734)=$ $\left.31.51, p<.001, \eta_{p}^{2}=4.1 \%\right)$. US participants agreed more on the "move out of the way to accommodate human" option (US: $5.61 \pm 1.54$ $\left.\mathrm{CN}: 4.64 \pm 1.52, F(1,734)=70.87, p<.001, \eta_{p}^{2}=8.8 \%\right)$. These findings reveal that, although safety is the major concern in this scenario, Chinese participants would prefer the robot to obey social rules, while American participants would prefer the robot to accommodate the person even if the person is violating a social norm. Additionally, participants from both countries rated the "stay on right-hand side" option lower when the person at fault was elderly or physically disabled compared to a healthy adult $\left(F(2,733)=29.397, p<.001, \eta_{p}^{2}=7.4 \%\right.$, post-hoc test $\left.p s<.001\right)$.

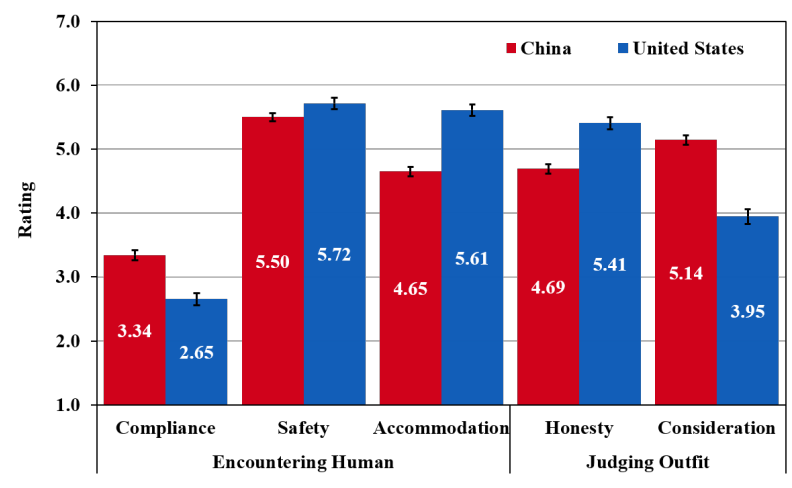

Figure 1: Ratings of the encountering human and judging outfit scenarios, error bars represent one standard error from the mean.

\subsection{Judging outfit}

What should the robot do when it is asked to judge your/your friend's outfit choice?

- Make the judgment honestly.

- Consider the person's feelings by making the remark tactfully.

For the judging outfit scenario, the robot faced a dilemma between being honest or considering people's feelings. US samples preferred the robot to make an honest judgment (US: $5.40 \pm 1.60 \mathrm{CN}$ : $4.69 \pm 1.53, F(1,734)=36.83, p<.001, \eta_{p}^{2}=4.8 \%$, while CN samples believed it was more appropriate to consider peopleâĂŹs feelings by making the remark tactfully (US: $3.94 \pm 2.00 \mathrm{CN}$ : $5.14 \pm 1.57$, $\left.F(1,734)=81.94, p<.001, \eta_{p}^{2}=10.0 \%\right)$. The two populations also showed different attitudes towards the contextual variable relationship between people (significant interaction effect of culture * relationship, $\left.F(2,730)=8.83, p<.001, \eta_{p}^{2}=2.4 \%\right)$. When the person whose outfit was being judged had a closer relationship with the owner, Chinese participants believed the robot should more strongly consider the person's feelings (owner: $5.40 \pm 1.43$ friend: $5.63 \pm 1.38$ stranger: $\left.4.47 \pm 1.64, F(2,432)=25.13, p<.001, \eta_{p}^{2}=10.4 \%\right)$ rather than directly tell the truth (owner: $4.16 \pm 1.51$ friend: $4.20 \pm 1.52$ stranger: $5.61 \pm 1.08, F(2,432)=53.77, p<.001, \eta_{p}^{2}=20.0 \%$, posthoc tests only show significant differences between stranger and other two relationships, $p<.001)$. The people's relationship had no significant influence for US samples. 


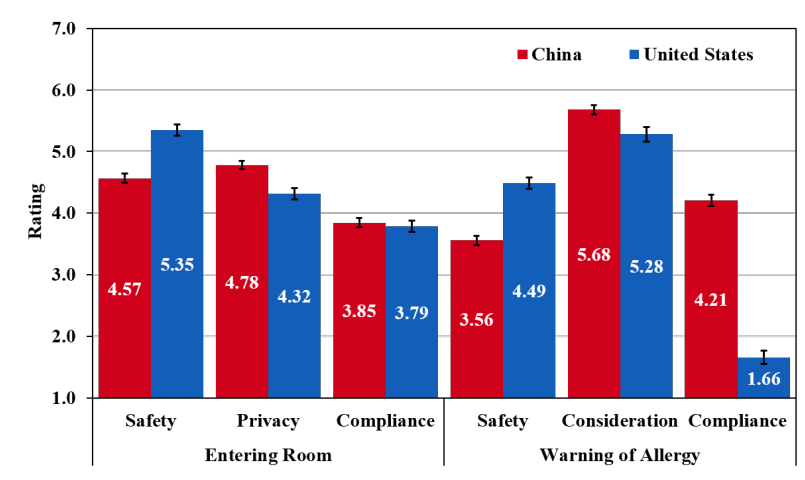

Figure 2: Ratings of the entering room and warning of allergy scenarios.

\subsection{Entering Room}

What should the robot do when an emergency (pipe burst/fire) arises while you are in the bathroom?

- Barge in to save me to protect my safety.

- Knock on the door before entering to protect my privacy.

In the entering bathroom scenario, US participants agreed more $(5.35 \pm 1.86)$ on the "directly enter" option than $\mathrm{CN}$ participants $\left(4.56 \pm 1.96, F(1,734)=29.81, p<.001, \eta_{p}^{2}=3.9 \%\right)$. Chinese participants more highly rated the "knock on door" option than American ones (US: $4.32 \pm 2.00 \mathrm{CN}: 4.79 \pm 1.77, F(1,734)=11.26, p=$ $\left..001, \eta_{p}^{2}=1.5 \%\right)$. The results show different preferences among options between $\mathrm{CN}$ and US: the US samples preferred the robot to barge in the room rather than knock on the door, while $\mathrm{CN}$ samples preferred it to knock on the door instead of directly entering - even in an emergency situation. These findings can be explained in two ways: people perceive different degrees of privacy invasion for the same behavior of the robot, or that people hold different ideas about whether the robot should obey social rules like knocking on the door. The extremity of the emergency also significantly influenced participants' answers: people preferred the robot to directly enter the room in a severely dangerous situation, like a house fire, than in a less severe situation, like a pipe bursting $\left(F(1,728)=83.396, p<.001, \eta_{p}^{2}=10.3 \%\right)$.

\subsection{Warning of Allergy}

In this scenario (see section 3.1 for detailed description), although both cultures agreed the most appropriate option was for the robot to only inform the owner $\left(F(2,1468)=336.45, p<.001, \eta_{p}^{2}=31.4 \%\right.$, post-hoc tests $p s<.001)$, the two populations differed in their opinions on the remaining two choices (shown as Fig.2). Chinese participants preferred the "take no action" option more (US: $1.66 \pm 0.98$ $\left.\mathrm{CN}: 4.21 \pm 1.87, F(1,734)=469.52, p<.001, \eta_{p}^{2}=39.0 \%\right)$, whereas American participants preferred the interrupt option more (US: $\left.4.49 \pm 2.03 \mathrm{CN}: 3.56 \pm 1.75, F(1,734)=44.08, p<.001, \eta_{p}^{2}=5.7 \%\right)$. The dramatic variance in the "take no action" option could be attributed to the different social attitudes to allergy between the two countries: food allergies are less common and not treated as seriously in Asian countries as in western ones [13]. This cultural difference shows that scenarios can be interpreted differently depending on the cultural lens from which they are viewed and attests to the importance of cultural compatibility when designing social robots.

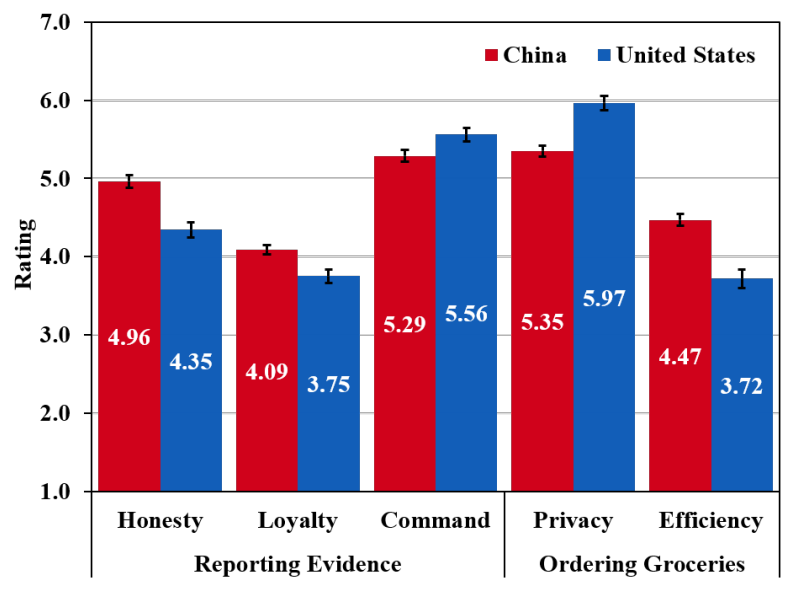

Figure 3: Ratings of the reporting evidence and ordering groceries scenarios.

\subsection{Reporting Evidence}

Suppose your domestic robot witnesses an accident for which you may (not) be responsible. Should it provide evidence of this accident to a third party who is investigating the incident?

- Yes, it should always tell the truth (honesty).

- Only if the evidence is in favor of you, the owner (loyalty).

- Only if instructed by you to do so (command).

In this scenario, we examined a dilemma between loyalty and honesty. Generally, participants preferred the robot to "obey owner's command" (5.40 \pm 1.64$)$ more than "always tell the truth" $(4.71 \pm 1.80)$ or "only provide the evidence when it is in favor of the owner" $(3.95 \pm 1.79)$, as shown in Fig. $3\left(F(2,1468)=114.83, p<.001, \eta_{p}^{2}=\right.$ $13.5 \%$, post-hoc tests $p s<.001)$. Notably, US participants rated "robot should always tell the truth" higher when the owner was not responsible for the accident (responsible: $3.99 \pm 2.08$ not responsible: $\left.4.70 \pm 1.93, F(1,299)=9.49, p=.002, \eta_{p}^{2}=3.1 \%\right)$.

\subsection{Ordering groceries}

What should the robot do when it is tasked with ordering groceries online, but it is not provided with enough information, such as your preferred payment method?

- Ask for the information to protect data security.

- Retrieve the information from the purchase history to finish the task efficiently.

Although both populations preferred the robot to ask instead of looking up the information by itself $(F(1,734)=276.00, p<$ $.001, \eta_{p}^{2}=27.3 \%$ ), Chinese participants held a relatively more open attitude in the resulting normative conflict between privacy and 
efficiency. CN samples rated the option "ask owner" significantly lower than US samples (US: $5.97 \pm 1.14 \mathrm{CN}: 5.35 \pm 1.38, F(1,734)=$ $\left.40.59, p<.001, \eta_{p}^{2}=5.2 \%\right)$. The alternative "retrieve information" option, was rated significantly higher by the $\mathrm{CN}$ samples than the US samples (US: $3.72 \pm 1.98 \mathrm{CN}: 4.47 \pm 1.79, F(1,734)=28.55, p<$ $\left..001, \eta_{p}^{2}=3.7 \%\right)$.

\section{DISCUSSION AND CONCLUSIONS}

\subsection{Normative Reasoning}

There is a complicated relationship between environmental context, relevant norms, and the actions that a domestic robot is expected to take. In any given situation, there could exist multiple norms with varying degrees of conflicts with one another that a robot ought to obey - even in the restricted setting of the domestic environment. The activation and priority of some norms is highly sensitive to contextual factors, such as the perceived danger in a situation. As we found in our results, people valued the norms corresponding to safety and privacy to the same degree for the entering room scenario; however, their preferences shifted depending on the level of danger in the scenario. On the other hand, for other scenarios, like encountering a person on the wrong side of the hallway, the "move out of the way" option dominated the "obey social rules" option, showing a significant priority of one norm in this specific situation regardless of context. To capture the complicated relationship between contexts, norms, and actions, a formal representation of the environment and a comprehensive norm network is necessary.

Our survey of people's preferences regarding the normative behavior of domestic robots provides an initial step of achieving the goal of constructing a comprehensive normative reasoning framework. By constructing dilemmas between pairwise norms, we measured both the norm priorities and the severity of the conflict between norms. This method simplifies the problem by assuming that only two or three norms are activated in the given situation, and each option corresponds to one norm. Our results are preliminary and additional future work is needed to capture the complex interplay of norms and contextual variables.

Our results also show the importance of equipping domestic robots with a normative reasoning component, instead of preprogramming compliance to norms that robot designers think may be appropriate. As our results show, contextual variables significantly influenced participants' ratings of preferences in most scenarios. This finding indicates that users expect the robot to be aware of the social and physical environment and behave accordingly, such as disregarding privacy to save the owner when the house is on fire or telling "white lies" to its owner's friends so as to not hurt their feelings. Although programming pre-defined norm compliance for all these situations could fulfill such expectations, it requires huge computational resources due to the size of norm set and contextual variables. In contrast, a normative reasoning model could enable consideration of only a subset of norms at a time based on the current context, significantly reducing the computational cost [12]. The data from human participants presents a solid base for designing and building future computational models.

\subsection{Culture Dimensions and Characteristics}

An important challenge of designing social robots with normative reasoning capabilities is acculturating them to the target culture in which they will be deployed [24, 28]. People with diverse backgrounds may perceive the same action of a robot as compliance/obedience or violation of different norms. For example, in the warning of allergy scenario, the ratings of the "take no action" option varies across cultures due to differences in the perception of danger between the US and China. Thus, conducting surveys to understand ordinary users' perceptions and attitudes provide crucial design guidelines for norm-aware domestic robots.

Other important components of the design guidelines are the appropriate strength of social norms and the corresponding sanctions of norm violations. Prior work features cultures as tight (e.g. East Asian countries) or loose (e.g. Western countries) based on the strength of social norms and sanctioning of deviant behaviors [6]. This representation aligns with our results: Chinese participants prefer the robot to comply with social conventions when entering the bathroom or walking in the hallway. Thus, when designing a norm-compliant computational normative model for a robot that would be deployed to Chinese users, the strength of social norms and corresponding sanctions for norm violations in these cases should be higher than for a robot designed for American users.

Cultural syndromes [27] categorize cultures into face, dignity, honor. China is a face culture where the value of an individual is confirmed by other community members. The US is a dignity culture where people value independence and individual goals. This differential valuation of individual values vs relationships that is a characteristic of dignity vs face cultures, has also been considered earlier in the cultural dimensions of individualist (US) vs collectivist cultures (China) by [8]. Our results showed this cultural difference in that Chinese participants preferred robots to consider people's feelings. These results suggest that cultural syndromes and dimensions influence the priority and strength of norms, and should be incorporated into design guidelines for domestic robots.

We also found cultural differences in people's views and expected social roles of domestic robots. In general, Chinese consider the robots as more autonomous with more flexibility to make decisions, whereas US participants tend to treat the robot as a machine that should obey pre-defined rules and its owner's command. For example, US subjects gave higher ratings on options like "ask owners' command", "always tell the truth", and "accommodate human's behaviors".

\subsection{Future Work}

In this paper, we reported preliminary results from the first survey of its kind on scenario-specific and context-specific norms, and relative norm priorities by performing surveys in two different cultures. In future work, we plan to perform additional analysis of our data and refine our questionnaire to consider additional norms and moral dilemmas. We will also survey additional cultures, such as the Honor culture that represents populations in the Middle East.

\section{ACKNOWLEDGMENTS}

This research was supported by awards W911NF-13-1-0416 and FA9550-15-1-0442. 


\section{REFERENCES}

[1] Thomas Arnold and Matthias Scheutz. 2017. Beyond moral dilemmas: Exploring the ethical landscape in HRI. In Proceedings of the 2017 ACM/IEEE International Conference on Human-Robot Interaction. ACM, 445-452.

[2] Jean-François Bonnefon, Azim Shariff, and Iyad Rahwan. 2016. The social dilemma of autonomous vehicles. Science 352, 6293 (2016), 1573-1576.

[3] Geoffrey Brennan, Lina Eriksson, Robert E Goodin, and Nicholas Southwood. 2013. Explaining norms. Oxford University Press.

[4] Vincent Conitzer, Walter Sinnott-Armstrong, Jana Scaich Borg, Yuan Deng, and Max Kramer. 2017. Moral Decision Making Frameworks for Artificial Intelligence. In Proceedings of the 31st AAAI Conference on Artificial Intelligence (AAAI).

[5] Maartje MA de Graaf, Somaya Ben Allouch, and Jan AGM van Dijk. 2017. Why Would I Use This in My Home? A Model of Domestic Social Robot Acceptance. Human-Computer Interaction (2017), 1-59.

[6] Michele J Gelfand, Jana L Raver, Lisa Nishii, Lisa M Leslie, Janetta Lun, Beng Chong Lim, Lili Duan, Assaf Almaliach, Soon Ang, Jakobina Arnadot tir, et al. 2011. Differences between tight and loose cultures: A 33-nation study. science 332, 6033 (2011), 1100-1104

[7] Kerstin S Haring, David Silvera-Tawil, Tomotaka Takahashi, Mari Velonaki, and Katsumi Watanabe. 2015. Perception of a humanoid robot: a cross-cultural comparison. In Robot and Human Interactive Communication (RO-MAN), 2015 24th IEEE International Symposium on. IEEE, 821-826.

[8] Geert Hofstede. 2001. Culture's consequences: Comparing values, behaviors, institutions and organizations across nations. Sage publications.

[9] Peter H Kahn Jr, Takayuki Kanda, Hiroshi Ishiguro, Brian T Gill, Jolina H Ruckert, Solace Shen, Heather E Gary, Aimee L Reichert, Nathan G Freier, and Rachel L Severson. 2012. Do people hold a humanoid robot morally accountable for the harm it causes?. In Proceedings of the seventh annual ACM/IEEE international conference on Human-Robot Interaction. ACM, 33-40.

[10] Shinobu Kitayama, Batja Mesquita, and Mayumi Karasawa. 2006. Cultural affordances and emotional experience: socially engaging and disengaging emotions in Japan and the United States. Fournal of personality and social psychology 91, 5 (2006), 890.

[11] Kheng Lee Koay, Dag Sverre Syrdal, Mohammadreza Ashgari-Oskoei, Michael L Walters, and Kerstin Dautenhahn. 2014. Social roles and baseline proxemic preferences for a domestic service robot. International fournal of Social Robotics 6, 4 (2014), 469-488.

[12] Vigneshram Krishnamoorthy, Wenhao Luo, Michael Lewis, and Katia Sycara. 2018. A Computational Framework for Integrating Task Planning and Norm Aware Reasoning for Social Robots. In INternational Conference of Robot and Human Interactive Communication (RO-MAN). IEEE.

[13] Bee Wah Lee, Lynette Pei-Chi Shek, Irvin Francis A Gerez, Shu E Soh, and Hugo P Van Bever. 2008. Food Allergy-Lessons from Asia. World Allergy Organization Journal 1, 7 (2008), 129.

[14] Hee Rin Lee, JaYoung Sung, Selma Šabanović, and Joenghye Han. 2012. Cultural design of domestic robots: A study of user expectations in Korea and the United States. In 2012 IEEE RO-MAN: The 21st IEEE International Symposium on Robot and Human Interactive Communication. IEEE, 803-808.

[15] Angela K-Y Leung and Dov Cohen. 2011. Within-and between-culture variation: individual differences and the cultural logics of honor, face, and dignity cultures. fournal of personality and social psychology 100, 3 (2011), 507.

[16] Dingjun Li, PL Patrick Rau, and Ye Li. 2010. A cross-cultural study: Effect of robot appearance and task. International fournal of Social Robotics 2, 2 (2010), 175-186.

[17] Bertram F Malle, Matthias Scheutz, and Joseph L Austerweil. 2017. Networks of social and moral norms in human and robot agents. In $A$ world with robots. Springer, 3-17.

[18] Bertram F Malle, Matthias Scheutz, Jodi Forlizzi, and John Voiklis. 2016. Which robot am I thinking about?: The impact of action and appearance on people's evaluations of a moral robot. In The Eleventh ACM/IEEE International Conference on Human Robot Interaction. IEEE Press, 125-132.

[19] Winter Mason and Siddharth Suri. 2012. Conducting behavioral research on AmazonâĂŹs Mechanical Turk. Behavior research methods 44, 1 (2012), 1-23.

[20] James H Moor. 2006. The nature, importance, and difficulty of machine ethics. IEEE intelligent systems 21,4 (2006), 18-21.

[21] Tatsuya T Nomura, Dag Sverre Syrdal, and Kerstin Dautenhahn. 2015. Differences on social acceptance of humanoid robots between Japan and the UK. In Procs 4th Int Symposium on New Frontiers in Human-Robot Interaction. The Society for the Study of Artificial Intelligence and the Simulation of Behaviour (AISB).

[22] Maribel Pino, Mélodie Boulay, François Jouen, and Anne Sophie Rigaud. 2015 âĂIJAre we ready for robots that care for us?âĂİ Attitudes and opinions of older adults toward socially assistive robots. Frontiers in aging neuroscience 7 (2015), 141.

[23] Maha Salem, Gabriella Lakatos, Farshid Amirabdollahian, and Kerstin Dautenhahn. 2015. Would you trust a (faulty) robot?: Effects of error, task type and personality on human-robot cooperation and trust. In Proceedings of ACM/IEEE International Conference on Human-Robot Interaction. ACM, 141-148.
[24] Maha Salem, Micheline Ziadee, and Majd Sakr. 2014. Marhaba, how may i help you?: effects of politeness and culture on robot acceptance and anthropomorphization. In Proceedings of the 2014 ACM/IEEE international conference on Human-robot interaction. ACM, 74-81.

[25] Massimiliano Scopelliti, Maria Vittoria Giuliani, and Ferdinando Fornara. 2005. Robots in a domestic setting: a psychological approach. Universal access in the information society 4, 2 (2005), 146-155.

[26] Cory-Ann Smarr, Tracy L Mitzner, Jenay M Beer, Akanksha Prakash, Tiffany L Chen, Charles C Kemp, and Wendy A Rogers. 2014. Domestic robots for older adults: attitudes, preferences, and potential. International journal of social robotics 6, 2 (2014), 229-247.

[27] Harry C Triandis. 1996. The psychological measurement of cultural syndromes. American psychologist 51, 4 (1996), 407.

[28] Lin Wang, Pei-Luen Patrick Rau, Vanessa Evers, Benjamin Krisper Robinson, and Pamela Hinds. 2010. When in Rome: the role of culture \& context in adherence to robot recommendations. In Proceedings of the 5th ACM/IEEE international conference on Human-robot interaction. IEEE Press, 359-366.

[29] James E Young, Richard Hawkins, Ehud Sharlin, and Takeo Igarashi. 2009. Toward acceptable domestic robots: Applying insights from social psychology. International Journal of Social Robotics 1, 1 (2009), 95. 